\title{
émulations
}

\section{Interdits alimentaires et aspects symboliques de la cuisine des Peuls du Ferlo (Sénégal)}

\section{Entre permanence, changement social et stéréotype}

\author{
Abdou Ka, Gilles Boetsch et Enguerran Macia
}

Émulations - Revue de sciences sociales

2019, Varia, En ligne.

\section{Article disponible à l'adresse suivante}

https://ojs.uclouvain.be/index.php/emulations/article/view/ka

\section{Pour citer cet article}

Abdou Ka, Gilles Boetsch et Enguerran Macia, « Interdits alimentaires et aspects symboliques de la cuisine des Peuls du Ferlo (Sénégal) : entre permanence, changements social et stéréotype », Émulations, Varia, En ligne. Mise en ligne le 29 août 2019.

DOI : 10.14428/emulations.varia.025

Distribution électronique : Université catholique de Louvain (Belgique) : ojs.uclouvain.be

(C) Cet article est mis à disposition selon les termes de la Licence Creative Commons Attribution, Pas d'Utilisation Commerciale 4.0 International. http://creativecommons.org/licenses/by-nc/4.0/

Éditeur : Émulations - Revue de sciences sociales / Presses universitaires de Louvain https://ojs.uclouvain.be/index.php/emulations

ISSN électronique : $1784-5734$

PUL PRESSES 


\title{
Interdits alimentaires et aspects symboliques de la cuisine des Peuls du Ferlo (Sénégal)
}

\author{
Entre permanence, changement social et stéréotype
}

Abdou Ka, Gilles Boetsch et Enguerran Macia ${ }^{1}$

\begin{abstract}
[Résumé] Les villages où nous avons recueilli des données sur les pratiques alimentaires se situent dans la zone sylvo-pastorale du Sénégal (Ferlo). Ce vaste territoire situé au nord du pays se caractérise par un climat semi-aride. Les projets de développement et de lutte contre la désertification s'y succèdent depuis les sécheresses des années 1970-80. La population est majoritairement peule, mais elle cohabite avec quelques familles wolofs et maures, ainsi qu'avec des agents de l'administration sénégalaise et d'ONG nationales et internationales. Ce travail de recherche vise à mettre en lumière le sens accordé aux interdits alimentaires et à la valeur symbolique de certains lieux et objets afférents à la cuisine des Peuls du Ferlo. L'étude de la retenue et des interdits alimentaires a fait émerger un fort sentiment d'appartenance à la culture peule, se distinguant - selon les habitants du Ferlo - par des valeurs et des normes morales contraires à l'exubérance caractéristique des autres ethnies. Notre analyse a également révélé les prémices d'un profond changement de la société du Ferlo, impactée par la globalisation et les migrations.
\end{abstract}

Mots clés : Peul, Ferlo, Sénégal, interdit alimentaire, changement social.

[Abstract] The villages where we collected food data are located in the silvopastoral zone of Senegal (Ferlo). This vast territory located in the northern part of the country is characterized by a semi-arid climate. Development and desertification control projects have been implemented there one after the other since the drought of the 1970s and 1980s. The majority of the population is Fulani but cohabitates with Wolofs and Moors, as well as Senegalese government officials and agents of national and international NGOs. This work aims at highlighting the meaning of the cultural dietary restrictions of the fulani people living in the Senegalese Ferlo region. We document the symbolic values held by particular tools and spaces related to the cooking in a context marqued by globalization and migration. The study of culinary restraint and food prohibitions brought to the foreground a strong feeling of membership in the Fulani culture and the beginnings of a deep change within the society of Ferlo.

Keywords: Fulani, Ferlo, Senegal, food ban, social change.

\footnotetext{
${ }^{1}$ Université Assane Seck de Ziguinchor, UMI ESS, Sénégal ; Faculté de Médecine, de Pharmacie et d'Odontostomatologie, UCAD, Sénégal ; Faculté de Médecine, de Pharmacie et d'Odontostomatologie, UCAD, Sénégal.
} 


\section{Introduction}

Chaque société est régie par des normes manifestes ou diffuses codifiant les pratiques alimentaires. Bon nombre de produits carnés comestibles, ou parfois végétaux comme chez les Polynésiens (Serra Mallol, 2012a), sont tabouisés² sans pour autant provoquer le dégoût ou la répulsion. Ces interdits alimentaires participent de la base identitaire de beaucoup de peuples et de religions. Chez les hindous, on note par exemple l'interdiction de manger de la viande de vache et, chez les juifs et les musulmans celle de manger de la viande de porc. Chez les chrétiens le manger "maigre » (sans viande ni graisse) est préconisé les vendredis et pendant le carême. Dans son ouvrage de référence Sociologies de l'alimentation, Jean-Pierre Poulain (2002) avance en substance que c'est à partir des thématiques du sacrifice, du totémisme et des interdits qui leur sont associés que les premiers ethnologues et sociologues ont appréhendé les faits alimentaires. C'est l'anthropologue Audrey Richards qui, dans les années 1930, se pose en véritable pionnière dans le domaine, à partir d'un terrain situé dans le sud-est de l'Afrique (en Rhodésie). Dans son premier ouvrage, intitulé Hunger and Work in a Savage Tribe, elle s'intéresse à l'organisation socio-économique des Bemba, aux tabous alimentaires, au totémisme et au sacrifice (Richards, 1932). Par la suite de nombreux auteurs ont observé des phénomènes d'interdits et de retenue alimentaires en Afrique subsaharienne comme en témoignent les travaux d'Igor de Garine chez les Wolofs et Sérères du Sénégal (1962), ceux de Pascal Zézé-Béké chez les Nyabwa de la Côte d'Ivoire (1989) ou encore ceux de Jean-François Gorre chez les Foulbé du Cameroun (1996).

Ce travail vise à mettre en lumière le sens accordé à la retenue, aux interdits alimentaires et à la valeur symbolique de certains lieux et objets afférents à la cuisine des Peuls en général et celle des Peuls du Ferlo en particulier. Ce peuple de pasteurs, réputé venir d'Orient, parcourt en permanence le Sahel ouest-africain à la recherche de pâturages et de points d'eau. Ahmadou Hampathé Ba les décrit en ces termes : «Les Peuls sont un surprenant mélange. Fleuve blanc au pays des eaux noires ; fleuve noir au pays des eaux blanches, énigmatique peuplement que de capricieux tourbillons ont amené du soleil levant et répandu de l'est en ouest presque partout»(Ba, 1991 : 21). D’après Maurice Delafosse (1972), les Peuls seraient originaires de la péninsule arabique. Pour Cheikh Anta Diop (1954), ils seraient venus d’Égypte. Selon Ahmadou Hampathé Ba et Germaine Dieterlen (1966), les Peuls trouveraient leurs origines dans le Sahara d'avant la désertification. Au Sénégal, quatre principaux foyers de peuplement peul ont été recensés. Tout d'abord au Fouta Toro, dans la vallée du fleuve Sénégal, se trouvent des populations peules, parfois métissées avec les populations environnantes, pratiquant l'agropastoralisme. Ces derniers ont été parmi les premiers propagateurs de l'Islam en Afrique de l'Ouest. Les Peuls Dieri occupent le centre et une partie du nord-est du pays.

2 De nombreux auteurs anglo-saxons ont étudié la notion de tabou à l'instar de M. Mead (1937), A. R. Radcliffe-Brown (1939) ou encore H. Webster (1942). 
Ce sont des éleveurs de zébus. Les Peuls du Kabada se retrouvent du côté de la Casamance, le long de la frontière gambienne. Enfin, les Peuls du Fouladou résident dans la région de Kolda. Ces deux derniers groupes sont plus ou moins influencés par la culture mandingue (Ka, 2013). Selon les discours des aînés rencontrés dans la région, les Peuls du Ferlo, venus de l'Est avec leurs troupeaux de bovins, d'ovins et de caprins, se sont tout d'abord implantés sur les rives sud du fleuve Sénégal pour profiter de l'abondance de ses eaux et des pâturages environnants $(\mathrm{Ka}, 2016)$. De là, ils quittèrent la vallée pour investir les hautes terres du Ferlo.

Le Ferlo, situé dans la zone sahélienne du Sénégal, s'étend sur les régions administratives de Saint-Louis, de Louga et de Matam. Il couvre une superficie de $57269 \mathrm{~km}^{2}$, soit $29 \%$ du territoire national (Demante, 2006). Aujourd'hui le Ferlo correspond à une zone sylvo-pastorale 3 . Cette dernière est délimitée au nord et à l'est par le fleuve Sénégal, à l'ouest par le lac de Guiers puis la vallée du Ferlo jusqu'à Linguère, et au sud par la route Linguère-Matam (carte 1).

Carte 1 : La zone sylvo-pastorale du Sénégal (source : O. Touré, 1997)

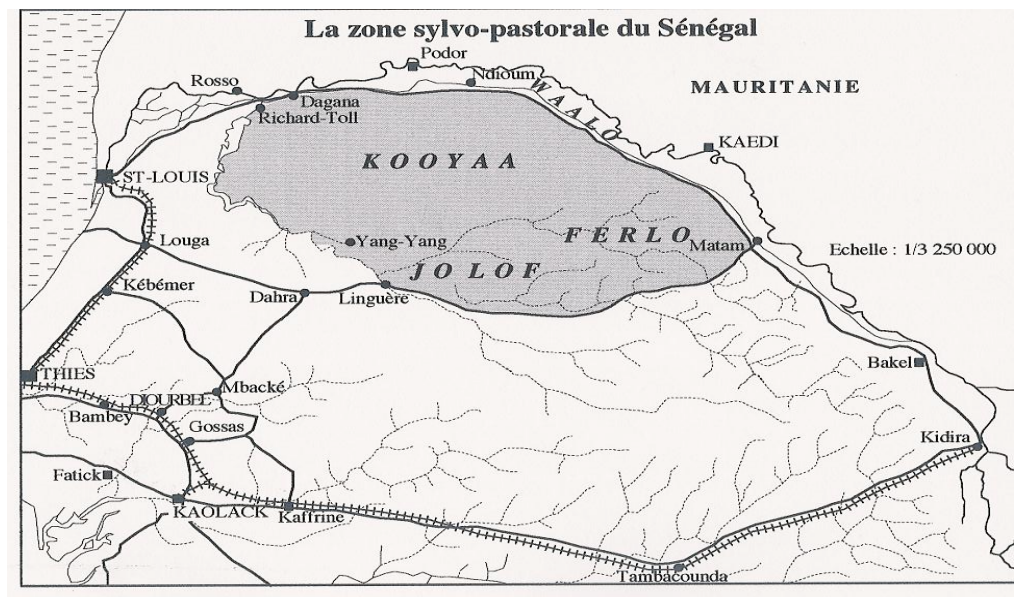

Depuis les années 1970, le paysage du Ferlo s'est progressivement transformé en se vidant peu à peu de ses plantes et de ses animaux, sous l'effet de la désertification et de l'augmentation des cheptels. Une enquête récemment menée dans la région a montré que les plus âgés se rappellent encore de la nature fertile et giboyeuse des années 1950 et 1960, où les Peuls cultivaient du mil et du niébé ${ }^{4}$ autour de leurs campements (Crenn, $\mathrm{Ka}, 2012$ ). La chasse, la cueillette des plantes alimentaires sauvages, l'agriculture et l'élevage permettaient aux Peuls de cette génération d'avoir une autonomie alimentaire.

\footnotetext{
${ }^{3}$ Une zone sylvo-pastorale est une vaste étendue géographique aménagée par l'État et réservée pour la promotion de l'élevage et de la foresterie.

${ }^{4}$ Le Vigna unguiculata est une plante légumineuse cultivée dans les campagnes sénégalaises pendant l'hivernage.
} 
Mais avec la désertification et la raréfaction des pluies, l'agriculture a été presque abandonnée et, depuis, les populations recourent aux marchés hebdomadaires pour s'approvisionner en denrées alimentaires. Cette dégradation de l'environnement dans cette partie du Sahel, à cause de facteurs climatiques et anthropiques, a également été décrite et analysée dans l'étude écologique de Michael Bollig et Anja Schulte (1999), corroborant le regard et la mémoire des plus âgés.

De nombreux projets de développement ont été menés dans cette zone. L'administration coloniale, tout d'abord, a entrepris le fonçage ${ }^{5}$ de plusieurs forages éparpillés dans la zone, dans le but de sédentariser les pasteurs peuls transhumants qui occupaient temporairement le Ferlo. Ces points d'eau permanents ont radicalement changé le mode de vie des pasteurs. Dans un second temps, d'importants projets de restauration d'une nature dégradée par la sécheresse de 1972-1973 ont été mis en place dans le cadre d'un vaste partenariat entre l'État du Sénégal et la communauté internationale ${ }^{6}$.

Pour l'instant, la plupart des études portant sur l'alimentation des Peuls se sont focalisées sur la figure atypique du Peul buveur de lait et vivant dans la brousse entouré de son bétail7. Elles n'ont pas beaucoup tenu compte des changements dus aux migrations $^{8}$, aux crises climatiques et à la globalisation. Or, de ces bouleversements a émergé ce que nous désignons comme une forme de « repli identitaire alimentaire » symbolisé par la forte revalorisation d'une valeur morale ancienne que les Peuls appellent $n d i-$ maagu. Le ndimaagu est un mode de vie caractérisé par des attitudes et des comportements de retenue, de simplicité, de sobriété et de frugalité. Peu étudiée par les anthropologues, cette valeur morale contraire à l'exubérance, qui caractérise pour les Peuls la majeure partie des groupes ethniques sénégalais, sera exposée dans les résultats de ce travail. La question liée aux restrictions que les Peuls s'imposent à eux-mêmes et surtout celle de la retenue alimentaire a, quant à elle, attiré l'attention de certains chercheurs. Salamata Sow considère que la retenue alimentaire est le code de comportement le plus remarqué par les étrangers en visite dans les sociétés rurales peules (Sow, 2015). Dans son ouvrage intitulé L'organisation sociale des Peuls, Marguerite Dupire (1970) nous apprend que l'un des traits caractéristiques de la culture peule est

\footnotetext{
${ }^{5}$ Le fonçage correspond à l'ensemble des activités nécessaires au creusement d'un forage (dans notre contexte).

${ }^{6}$ BARRAL H. (1982), « Le Ferlo des forages. Gestion ancienne et actuelle de l'espace pastoral », article disponible sur: http://horizon.documentation.ird.fr/exl-doc/pleins_textes/divers1506/010004749.pdf, consulté le 15 juin 2015.

${ }^{7}$ Ba S.-M, (2009), "Entre les pasteurs peuls et leurs vaches : une "parenté de lait" », article disponible sur: http://www.lemangeur-ocha.com/wp-content/uploads/2012/04/Le-lait-desPeuls.pdf, consulté le 22 octobre 2013 ; D. Guilhem, (2006), « Le lait de vache dans les sociétés peules. Pratiques alimentaires et symbolisme d'un critère identitaire », article disponible sur: http://www.lemangeur-ocha.com/texte/le-lait-des-vaches-dans-les-societes-peulespratiques-alimentaires-et-symbolisme-dun-critere-identitaire/, consulté le 30 décembre 2015.

${ }^{8}$ Notamment à l'exode rural et aux retours temporaires des (nouveaux) urbains.
} 
l'importance des attitudes d'évitement qui sont régies par un code socio-moral appelé pulaaku, qui signifie, selon elle, la manière de se comporter en peul. Les propos d'Oumarou, un Peul, rapportés par Henri Bocquené vont dans le même sens :

Quand partis très tôt le matin à jeun, nous arrivons chez quelqu'un tard le soir sans avoir mangé quoi que ce soit, il nous faut faire preuve d'une grande maîtrise de nous-mêmes. Nous ne devons manifester ni notre fatigue ni notre faim. On ne peut que toucher du bout des doigts à ce qui est apporté, deux ou trois bouchées seulement. II faut résister énergiquement aux invitations pressantes que les gens se croient obligés de faire. (Bocquené, 1986 : 313)

Roger Labatut (1973) précise que le pulaaku inclut les grandes qualités morales peules (le jugement, la patience, le souci des autres, la réserve), mais également des règles de politesse plus conventionnelles comme le fait de ne pas manger en public.

Cette image d'Épinal du Peul vivant en vase clos tout en gardant ses valeurs ancestrales intactes est loin de refléter la réalité concrète des habitants du Ferlo sénégalais. Ces pasteurs transhumants subissent les mutations occasionnées par la circulation d'idées, de marchandises et d'humains dans un cadre globalisé où personne n'échappe aux effets des innovations technologiques (téléphonie, télévision, panneaux solaires, etc.). Au travers du prisme de l'alimentation, nous chercherons à comprendre comment les Peuls du Ferlo combinent ces multiples références tout en préservant leur identité culturelle. Partant de l'étude anthropologique des restrictions et des interdits alimentaires, l'ambition de cet article est de déconstruire certains stéréotypes concernant les Peuls en décrivant les pratiques contemporaines et de proposer des pistes de réflexion sur ces transformations.

\section{Matériel et méthode}

De juillet 2012 à août 2015, le premier auteur de cet article a parcouru le Ferlo pour y mener des observations et des entretiens concernant les pratiques alimentaires des populations'. Cette enquête en anthropologie de l'alimentation tend à envisager le mangeur comme un individu social, qui ne peut être détaché du contexte dans lequel il évolue. Celui-ci ne saurait être réduit à son seul statut d'être biologique mangeant pour survivre, ni encore à son statut de consommateur. Mais ces deux facettes du mangeur sont néanmoins prises en compte dans l'analyse. Dans cette étude nous avons fait usage d'une méthode qualitative, basée sur des entretiens semi-directifs, complétés par des observations fines et détaillées des différents espaces investis (bourgs,

\footnotetext{
${ }^{9}$ Remerciements : ce travail a bénéficié d'une aide de l'État français gérée par l'Agence Nationale de la Recherche au titre du LabexDRIIHM, programme "Investissements d'avenir » portant la référence ANR-11-LABX-0010 et d'un soutien du projet « Milkyway » (financé par la Mission pour l'Interdisciplinarité du CNRS).
} 
campements $^{10}$, forages, marchés hebdomadaires, gargotes, jardins polyvalents, etc.). Les notes consignées dans les carnets de terrain lors des observations constituent des données qui ont permis d'étoffer et d'affiner les analyses issues des entretiens. Le guide d'entretien mis en place pour répondre à notre problématique a permis d'aborder avec les interviewés des questions liées aux restrictions, puis aux représentations alimentaires, afin d'éviter toute posture réifiante de la culture alimentaire de cette zone.

L'échantillonnage de convenance et la méthode de la boule de neige ${ }^{11}$ (Combessie, 2007) nous ont permis d'élargir notre population d'enquête afin d'obtenir un échantillon diversifié. Au total, treize villages ont été étudiés en profondeur. Des individus résidant dans d'autres villages ont par ailleurs été interviewés au gré des opportunités : après une réunion dans les locaux des Eaux et forêts ${ }^{12}$, au marché hebdomadaire, après une visite au poste de santé, etc. La grande majorité des personnes interrogées résidaient dans le Ferlo - principalement dans les campements - mais quelques commerçants colporteurs ont également été interviewés.

\section{Le ndimaagu : une morale alimentaire valorisée}

En règle générale, les populations de pasteurs transhumants que nous avons rencontrées présentent une alimentation frugale et peu diversifiée. Les repas servis aux campements sont la plupart du temps du riz au niébé. Ce mets est composé de riz, d'huile végétale, de niébé (petits haricots rouges ou blancs) et de poisson fumé et/ou séché. Cette cuisine simple est due à un problème de disponibilité et de conservation de certains ingrédients. Les habitants des campements ne peuvent pas se procurer chaque jour des produits frais à cause de la longue distance qui sépare les campements et les bourgs. Dans les discours recueillis, la frugalité est socialement valorisée et elle est considérée comme un marqueur de l'identité. La notion de ndimaagu permet d'expliciter le sens que cette société octroie à la frugalité et à la sobriété. Contrairement au pulaaku qui est partagé par la quasi-totalité des Peuls, sans occulter l'existence de nuances dans son interprétation, le ndimaagu est réservé aux Peuls dimo, à savoir les Peuls qui se considèrent comme étant « libre » et «noble». Les membres des castes (forgerons, bijoutiers, tisserands, boisseliers, etc.) et descendants de captifs ne sont pas censés suivre les règles du $n$ dimaagu ${ }^{13}$. Dans son ouvrage Peul du Ferlo, le voyageur et homme politique Philippe Grenier relevait déjà qu'« au dimo, l'homme libre, s'opposent le mathioudo, l'esclave, et le laobé, celui qui travaille le bois » (Grenier, 1960 : 38). Offrant un

\footnotetext{
${ }^{10}$ Les campements sont des habitations formant de petits villages satellites aux bourgs créés à côté des forages.

${ }^{11}$ La boule de neige consiste à entrer en contact avec de nouveaux enquêtés par l'intermédiaire des personnes interrogées précédemment.

12 La Direction des Eaux et Forêts, Chasses et de la Conservation des sols est sous la tutelle du Ministère de l'environnement. Elle est chargée de la protection des ressources naturelles. ${ }^{13}$ Dans notre zone d'étude, les castes et les descendants de captifs constituent une minorité peu visible.
} 
constat similaire, les anthropologues Anneke Breedveld et Mirjam De Bruijn (1996) estiment que trois composantes essentielles caractérisent le comportement des pasteurs peuls du Centre-Mali : ndimu (noblesse), yaage (retenue) et juulde (piété). Elles précisent que celui qui applique ces trois règles est considéré comme un Pullo dimo.

Le ndimaagu peut être défini comme un ensemble de normes, de pratiques, de valeurs, d'attitudes sociales, de comportements, de modes vestimentaires et alimentaires que le Peul dimo est supposé adopter en toutes circonstances. Au Ferlo, nos interlocuteurs nous ont énuméré les principales caractéristiques du ndimaagu :

- $\quad$ un Peul peut avoir faim et décliner une invitation à manger à cause du ndimaagu ;

- le ndimaagu interdit de manger un plat déjà entamé ;

- $\quad$ le ndimaagu interdit de finir un repas ;

- le ndimaagu interdit de parler beaucoup ;

- le ndimaagu interdit de porter des habits excentriques ;

- le ndimaagu incite à se contenter de peu.

La moitié des lignes de conduite édictées par le ndimaagu $u^{14}$ concerne spécifiquement l'alimentation et vient éclairer le rapport ascétique que ces populations entretiennent avec elle au quotidien.

Le ndimaagu peut être analysé comme le soubassement moral ou idéologique de la vie austère qu'ils mènent et prônent. Il participe également à la construction de leur identité, ou d'une frontière culturelle (au sens de Frederick Barth, 1969) avec l'exogroupe. Comme en témoignent ces propos d'un homme de 53 ans: "Le Peul c'est quelqu'un de timide. C'est pourquoi il ne peut pas manger en présence des étrangers. Il va se sentir gêné. Il peut avoir faim et préférer ne pas manger s'il n'est pas chez lui. Il est éduqué ainsi ». Cette idéalisation du ndimaagu par les Peuls du Ferlo n'est toutefois pas étrangère au contexte actuel caractérisé par un manque alimentaire et par un processus de différenciation identitaire face aux voisins wolofs et aux chercheurs et agents de développement passant dans la région. En effet, les Peuls du Ferlo se réfèrent au ndimaagu pour faire face aux autres groupes ethniques sénégalais qui les considèrent comme peu offrants, avares, au point de ne pas profiter de leurs biens (comme le bétail). Comme le rappelle le témoignage d'un habitant du Ferlo âgé de 67 ans : « Le Peul ne mange pas beaucoup. C’est ce mode d'alimentation qui est meilleur pour sa santé. Il faut toujours modérer son alimentation. Même avec de l'eau, il faut limiter la consommation ». Phillipe Grenier avait déjà observé ce comportement ascétique chez les Peuls du Ferlo, sans faire référence à la notion de ndimaagu. Il l'avait davantage analysé comme une forme d'abandon de soi : « ils négligent de se nourrir, mangent peu et n'importe quand; ou même ils ne mangent pas du tout quand ils suivent le troupeau en brousse et au puits »(Grenier, 1960 :54). Dorothée Guilhem (2012) a retrouvé une notion similaire au ndimaagu chez les Peuls de Senossa au Mali : le yaage. Cette valeur morale impose au Peul une endurance à la faim et à la soif, au même titre qu'elle codifie les

\footnotetext{
${ }^{14}$ Le ndimaagu est transmis aux jeunes générations par les pasteurs les plus âgés à travers leurs comportements quotidiens ou par l'oralité (contes, maximes, dictions).
} 
manières de table. Elle sert également de distinction entre les Peuls «libres » (fulbe) et les descendants de captifs (riimaybe), ces derniers étant considérés par les premiers comme dépourvus de yaage.

\section{Autour du repas : un moment de socialisation alimentaire}

Au moment des repas, nous avons observé une organisation sociale bien spécifique autour du plat commun, revêtant des distinctions d'âge, de genre et de statut. Les femmes mangent à part avec les jeunes et petites filles, et avec les garçons âgés de moins de cinq ans. À partir de cet âge, les garçons sont introduits à la table des hommes, qui partagent leur repas avec le chef du campement. Pendant le repas, la personne la plus âgée de chaque groupe veille sur les enfants et leur apprend à bien se comporter. Ceux-ci sont sommés de bien tenir le rebord du bol par un doigt de leur main gauche. Ils ne doivent ni parler, ni regarder les adultes. "Quand je mange avec les enfants j'en profite pour les corriger parce qu'un enfant ne doit pas manger en regardant les adultes. Il doit baisser la tête. » (propos d'un homme interviewé d'une soixantaine d'années)

Certaines injonctions décrites dans l'ouvrage d'Amadou Hampathé Ba, Amkoullel, l'enfant peul (1991), et apprises aux enfants dès leur plus jeune âge sont similaires à celles que nous avons ethnographiées dans les villages du Ferlo. Selon l'auteur, les enfants sont soumis à une discipline rigoureuse durant le repas. Ceux qui y manquent sont punis et font l'objet, selon la gravité de leur faute : d'un regard sévère, d'un coup d'éventail sur la tête, d'une gifle, voire même d'un renvoi pur et simple avec privation de nourriture jusqu'au repas suivant. Voici les sept règles impératives ${ }^{15}$, rapportées par Amadou Hampathé $\mathrm{Ba}$, que les enfants peuls doivent observer au moment du repas :

- ne pas parler;

- tenir les yeux baissés durant le repas;

- manger devant soi (ne pas grappiller à droite et à gauche dans le grand plat commun);

- ne pas prendre une nouvelle poignée de nourriture avant d'avoir terminé la précédente ;

- tenir le rebord du plat de la main gauche;

- éviter toute précipitation en puisant la nourriture avec sa main droite ;

- $\quad$ enfin, ne pas se servir soi-même les morceaux de viande déposés au centre du grand plat.

Amadou Hampathé Ba avance que les enfants doivent se contenter de prendre des poignées de céréales (mil, riz ou autre) bien arrosées de sauce ; il précise que ce n’est qu’à la fin du repas qu'ils reçoivent une pleine main de morceaux de viande considérée

\footnotetext{
${ }^{15}$ Les six premières règles citées par $\mathrm{A}$. $\mathrm{H}$. Ba sont toujours pratiquées au moment des repas dans le Ferlo mais nous n'avons pas pu observer la dernière. D'après nos observations, les adultes n'émettent aucune objection à ce que les enfants prélèvent du poisson ou de la viande au milieu du grand plat.
} 
comme un cadeau ou une récompense. Il explique que cette discipline ne vise nullement à faire souffrir l'enfant, mais à lui enseigner un art de vivre (Ba, 1991). Au Ferlo, les consignes imposées aux enfants au moment du repas servent à leur apprendre à bien se comporter dans la société. Les règles de bienséance qu'A. H. Ba a apprises pendant son enfance lors des repas sont toujours prégnantes chez les pasteurs peuls du Ferlo. Manger n'est pas seulement un acte servant à nourrir l'organisme mais constitue un moment de formalisation et de transmission des règles de vie en société, un moment où, à la fois on mange et on apprend à devenir « un bon Peul ».

Chez les enfants wolofs du Sénégal, Jacqueline Rabain-Jamin (2003) a observé une autre forme de socialisation dans laquelle les adultes se retrouvent aussi impliqués : «La conversation de l'adulte avec l'enfant s'effectue sur le modèle de questions/réponses qui s'enchaînent de manière rythmée et ont pour thème récurrent des activités socialement pertinentes (préparer le repas, vendre ou acheter, accompagner un hôte, etc.) » (p. 49). L'existence de codes moraux comme le pulaaku et le ndimaagu n'est pas une spécificité peule. À titre d'exemple dans la société sénégalaise, la plupart des groupes ethniques mettent en avant une variabilité de valeurs à l'instar de la pudeur, la discrétion, le courage, la loyauté, qui, inscrits dans la mémoire collective, reviennent à l'attention et au goût du jour à travers les chansons, les contes ou, plus prosaïquement, les réprimandes au quotidien (Camara, 2013). Le terme jom signifie en wolof à la fois le courage, l'abnégation et le contrôle de soi ; celui de teranga se traduit par l'hospitalité (Ly, 1966). Compte tenu de l'importance de la teranga, la commensalité vise à mettre les invités à l'aise en leur offrant le meilleur repas possible. La présentation du repas dans un plan commun facilite donc la réalisation de l'obligation sociale d'y convier tous les visiteurs (Yount, 2010).

\section{Une cuisine interdite aux hommes}

Outre les règles qui régissent le moment du repas et les interdits alimentaires, il en est d'autres qui concernent l'espace culinaire. Au Ferlo, la « cuisine » se trouve toujours en bordure des campements. La première raison fournie par nos interlocuteurs et interlocutrices quant à cette position excentrée est celle d'éviter les feux de brousse. De prime abord, cette explication fonctionnelle est plausible puisque la plupart des campements sont faits de bois et de paille, et un éventuel incendie pourrait occasionner d'importants dégâts. En approfondissant les entretiens, nous avons cependant pu constater que cette mise à distance de la cuisine concorde pleinement avec l'évitement dont doivent faire preuve les cuisinières pour ne pas laisser une marmite au milieu de la cour ou à proximité de la case du chef de campement. Ce n'est qu'au décès de ce dernier que la marmite et la hache peuvent, et doivent, être placées devant sa case. Les habitants voient dans ces modes de faire des " traditions » anciennes qu'ils ont héritées de leurs parents. Toutefois ces pratiques ont d'après nos observations tendance à disparaître avec les nouvelles générations. Au Ferlo, les plus jeunes aspirent à plus de « modernité » 
(notamment par l'adoption du mode de vie urbain) comme le démontre par ailleurs l'étude de Claire Manoli et Véronique Ancey (2013). Le travail de ces chercheuses révèle que le modèle du pasteur vivant en brousse de façon frugale, sorte d'idéal prôné par les chefs de famille, n'est pas toujours choisi par les plus jeunes.

Cette difficulté à mettre en relation les ustensiles de cuisine avec la disparition du chef du campement aiguise la curiosité du chercheur tout en montrant les limites de la recherche sur les interdits. C'est pourquoi Luce Giard (1994) affirme que s'il est difficile de mettre en évidence la cohérence du système d'interdits, c'est que l'efficacité des processus symboliques semble être garantie par leur caractère inconscient, soutenu d'une justification défensive et d'un refus d'entrer plus en avant dans la discussion.

\section{Photo 1: Une cuisine peule dans un campement du Ferlo (A. Ka, 2014)}

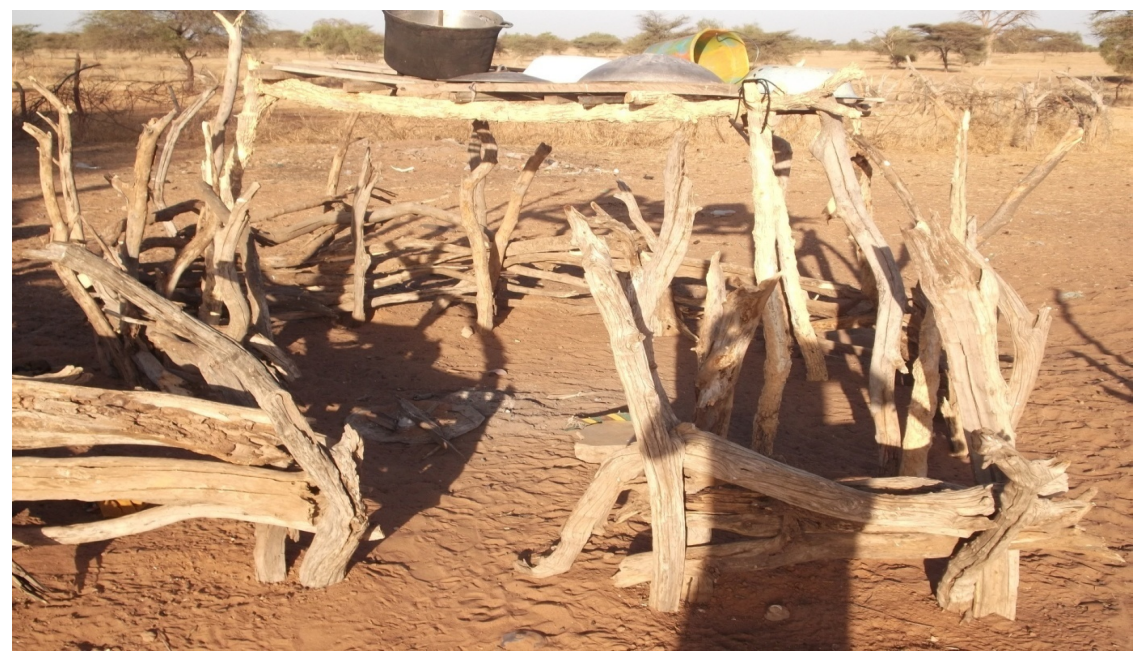

Dans les campements, les ustensiles de cuisine sont rangés dans les cases. Précisons qu'aujourd'hui, toutes les cases ne sont plus faites uniquement de bois et de paille : certaines sont fabriquées en ciment et des toits en zinc sont apparus. Mais la forme initiale reste toujours la même. La cuisson des aliments est réservée aux femmes et est considérée comme une activité strictement féminine. Certains hommes refusent même de toucher les marmites parce que cela pourrait altérer l'efficacité des amulettes qu'ils portent sur eux ${ }^{16}$. La marmite, selon les personnes que nous avons interviewées, est

${ }^{16}$ Les amulettes sont des objets fabriqués par les guérisseurs et qui prennent la forme de
pendentifs, d'anneaux, ou de cordelettes. Elles sont constituées généralement de morceaux
de cuir, de végétaux, de corne ou d'os. Elles sont supposées attirer la chance et protéger les 
porteuse d'une charge symbolique très négative capable de détruire des forces surnaturelles bénéfiques. D'aucuns ne la gardent dans leurs cases. Après le repas, la marmite est suspendue sur le toit de la cuisine ou sur la hutte ${ }^{17}$ qui sert d'ombre au campement. Dans ces situations, nous sommes ici face à des phénomènes de contamination négative, où le contact avec la marmite produirait un effet désacralisant ou "polluant » (Douglas, 1971) sur les objets sacrés ou chargés d'une force surnaturelle (autel, grigri, talisman, amulette, etc.). Chez les Polynésiens, ce phénomène de contamination négative a également été mis en évidence : lorsqu'un homme sur le point d'être sacrifié réussissait à se faire mordre par une femme, et que son sang coulait, il devenait impropre au sacrifice et sauvait ainsi sa vie (Serra Mallol, 2012b). Au Ferlo, cette contamination négative survient par simple proximité, elle peut se réaliser à distance. Selon les hommes, passer au-dessus d'un pilon qui sert à écraser des céréales ou des feuilles dans le mortier annihile les pouvoirs magico-religieux de la personne et la rend vulnérable aux esprits malveillants, comme aux armes blanches ${ }^{18}$. Cette gestion genrée de l'espace domestique sous-tendue par un ensemble d'interdits justifie le cantonnement des femmes à la cuisine et libère, en conséquence, les hommes de certaines tâches ménagères harassantes, parmi lesquelles la préparation des repas et du lait, de même que les corvées d'eau. Au-delà de la division sexuelle des tâches domestiques, la peur manifeste des hommes d'être contaminés par des objets culinaires « féminins » révèle la manière dont les pasteurs du Ferlo perçoivent l'être humain et ses rapports à un ordre social et surnaturel. Cette conception cosmologique rend intelligible une fabrique différenciée des genres et de leurs rôles sociaux. Mais l'apparent confinement des femmes à la cuisine ne doit pas occulter l'émergence de formes de leadership féminin dans ce territoire marqué par les soubresauts de la globalisation et les réformes étatiques.

\section{L'émergence d'une élite féminine et de nouvelles normes sociales}

Depuis l'avènement des projets de développements et des ONG, vers la fin des années 1970, œuvrant pour la restauration d'une nature dégradée par la crise climatique et contre la pauvreté, les femmes connaissent des formes visibles d'émancipation. Se met ainsi en place depuis vingt ans une véritable élite féminine, dont le fait que

gens des attaques (physiques et sorcellaires) de personnes malveillantes ou d'esprits maléfiques.

${ }^{17}$ La hutte sert à créer de l'ombre au sein du campement et constitue par conséquent le lieu de convivialité où se déroulent les discussions.

${ }^{18}$ Certains pasteurs peuls du Ferlo consomment une bouillie de mil ou de riz mélangée avec une potion appelée tul permettant de rendre imperméable la peau aux attaques à l'arme blanche. 
l'actuelle cheffe du gros village de Widou Thiengoly ${ }^{19}$ soit une femme constitue un exemple. Ceci est un évènement relativement inédit dans cette société encore largement patriarcale. Les groupements de promotion féminine sont soutenus par des mutuelles de crédit et d'épargne, ainsi que par le projet de la Grande Muraille verte. Les jardins polyvalents du projet de la Grande Muraille verte sont gérés uniquement par des organisations de femmes (Ka, 2013 ; Billen, 2014). Les agents de ce projet s'appuient sur des groupements de femmes pour faire fonctionner les potagers et les vergers. Avec l'appui de leurs partenaires (Food \& Agriculture Organisation, Programme alimentaire mondial, etc.), les femmes ont la possibilité de suivre régulièrement des formations en horticulture, en gestion des dividendes, en restauration par des techniciens qualifiés déployés sur le terrain. La Grande Muraille verte fournit les semences, le matériel et le gasoil pour le pompage de l'eau à partir des forages jusqu'aux périmètres cultivés. Les témoignages de cette femme ${ }^{20}$, impliquée dans le fonctionnement du jardin polyvalent, sont du même ordre d'idées :

La récolte est d'abord achetée par les femmes du groupement. Parfois elle ne nous suffit pas. Les agents de la Grande Muraille verte en achètent aussi. Ils disent que les pommes de terre et les oignons sont de bonne qualité. On garde l'argent de la vente de nos produits dans nos caisses. Chaque femme a la possibilité d'en emprunter et de nous rembourser avec un taux d'intérêt de $10 \%$. (Femme, 43 ans)

Ces actrices du changement deviennent à présent des interlocutrices privilégiées des bailleurs de fonds et des autorités administratives et politiques ${ }^{21}$. Être membre d'un groupement des femmes qui s'occupe des jardins polyvalents est aussi un moyen de pouvoir bénéficier des dons du PAM, permettant un échange de vivres (maïs, petits pois, riz, sel iodé, huile de cuisine, etc.) contre du travail dans les villages du Ferlo où les plantations de la Grande Muraille Verte sont mises en place. Intégrées dans ces activités de développement local elles veulent investir d'autres créneaux comme l'aviculture, l'apiculture, la création d'unités de transformation de lait pour augmenter leur pouvoir d'achat.

Depuis 2014, au Sénégal les conseillers municipaux sont pour moitié des femmes suite à la promulgation de la loi du 19 mai 2010 sur la parité homme-femme dans les instances totalement ou partiellement électives. Auparavant dans notre zone d'étude aucune femme n'avait jamais siégé au Conseil municipal. Nous assistons donc ici à une plus grande implication formelle des femmes dans la gestion du territoire du Ferlo. Le

\footnotetext{
${ }^{19}$ En 2015, Widou Thiengoly comptait 6165 habitants d'après le recensement de Margaux Mauclaire (Thèse de géographie en cours, Université Bordeaux 3 Montaigne.

${ }^{20}$ Chaque jardin polyvalent de la Grande Muraille Verte est géré par un groupement de 200 à 250 femmes.

${ }^{21}$ Des pratiques semblables de renforcement de l'empowerment des femmes ont été observées notamment par Isabelle Droy au Mali avec la mise en place d'une filière de coton biologique sous l'impulsion d'une ONG suisse (Droy, 2011).
} 
travail de Pierre Michaud et Nacuzon Sall (2005) a démontré que dans plusieurs pays du Sahel, des projets ont tenté d'offrir des programmes d'éducation de base aux femmes et aux décrocheurs de l'école publique dont les éléments constituants dépassent le simple apprentissage du savoir lire, écrire et compter, et concernent la maîtrise d'aptitudes sociales, civiles et l'acquisition de certains métiers et de compétences de base. Ces femmes-leaders sont au cœur de la diffusion des normes sociales et alimentaires dans le Ferlo. Dans les deux parties subséquentes nous allons mettre en exergue d'autres éléments importants tels les interdits alimentaires, la religion ou une origine lointaine qui ont aussi permis aux Peuls de marquer leur identité et de créer une frontière culturelle vis-à-vis des autres groupes ethniques sénégalais.

\section{5. « Vous ne mangerez point de leur chair... "}

La consommation de chair animale est sujette à de nombreux interdits et règles explicités dans des textes religieux: par exemple le "manger » kasher chez les juifs ou le «manger » halal chez les musulmans. Très tôt et dans la plus grande diversité des cultures, des frontières entre le comestible et l'incomestible ont pris forme en fonction des expériences vécues ou des représentations sociales. Pour les Peuls du Ferlo, la consommation de la chair de lièvre ou de perdrix constitue un interdit et peut occasionner la perte future du bétail. Chez les Juifs, un certain nombre d'animaux sauvages, semblables aux animaux domestiques demeurent propres à la consommation; ceux qui ont le sabot fendu et qui ruminent. Mais les lièvres et les lapins n'en font pas partie (Farb, Armelagos, 1980). L'observance des interdits alimentaires religieux est un élément ancien de l'identité culturelle des Peuls. Très tôt ils ont été convertis à l'Islam par leurs voisins arabo-berbères, qui ont été parmi les principaux relais de l'Islam au sud du Sahara (Schmitz, 1990).

Pourtant, la chair de ces animaux est très recherchée par beaucoup d'autres groupes ethniques du Sénégal. En effet, les interdits alimentaires sont réactualisés, différemment négociés, voire renforcés au grès des situations sociales et des rapports « interethniques » contemporains. Selon Claude Fischler, la variabilité des choix alimentaires procède pour une grande part de la variabilité des systèmes culturels. Il estime que si nous ne consommons pas tout ce qui est biologiquement comestible, c'est que tout ce qui est biologiquement mangeable n'est pas culturellement comestible (Fischler, 2001). Pour les habitants du Ferlo, la chair des bucorves d'Abyssinie est considérée comme toxique à cause des scorpions et des grenouilles dont ces grands oiseaux se nourrissent. Ce phénomène de contamination par analogie a déjà été observé chez les Bororos en Amazonie brésilienne par Claude Lévi-Strauss (1962). 


\section{La symbolique du kaggu et des calebasses contenant le lait}

Pendant l'hivernage, la vente des produits laitiers constitue la principale activité génératrice de revenus pour les femmes. La commercialisation du lait et des laitages est le fait des femmes au même titre que celle du bétail l'est pour les hommes. Cependant la conservation du lait requiert une organisation minutieuse. Dans la case de chaque épouse sont disposées deux sortes de tables semi-circulaires appelées kaggu. Elles sont installées l'une à droite et l'autre à gauche du lit conjugal. Sur le premier kaggu, celui de droite en entrant, sont posées les calebasses qui contiennent le lait, et sur le deuxième kaggu sont disposés les ustensiles de cuisine (écumoires, louches, bols, couscoussier, etc.). Sur le kaggu de droite peuvent également être posés les sacs de mil et de riz. Dans les cases en ciment, les kaggu sont faits en dur. Selon une femme peule rencontrée dans un campement : « le kaggu c'est la tradition peule. Avant on allait chercher du bois dans les bas-fonds pour construire de très belles cases. Et à l'intérieur on construisait deux kaggu. On y gardait le lait et le mil. On n'utilisait pas les bols en métal. Il n'y avait que des calebasses ». L'utilisation des calebasses est toujours présente dans les campements que nous avons visités. Le largal est la plus grande et la première calebasse du kaggu. On y met le lait frais avant de le transvaser dans les autres calebasses. Au sens figuré, le largal renvoie à l'homme qui illumine les consciences. C'est la personne intelligente et instruite qui partage ses connaissances avec les autres. Les autres calebasses s'appellent petdirndé (celles qui servent à cailler le lait). Elles contiennent le walndé et le nialoundé. Le walndé est le lait qui est trait le soir et gardé jusqu'au lendemain. On récupère sa crème vers 14 heures. Le nialoundé est le lait du matin. On récupère sa crème le lendemain matin.

Photo 2 : Un kaggu avec des calebasses en bois sculpté (A. Ka, 2015) 


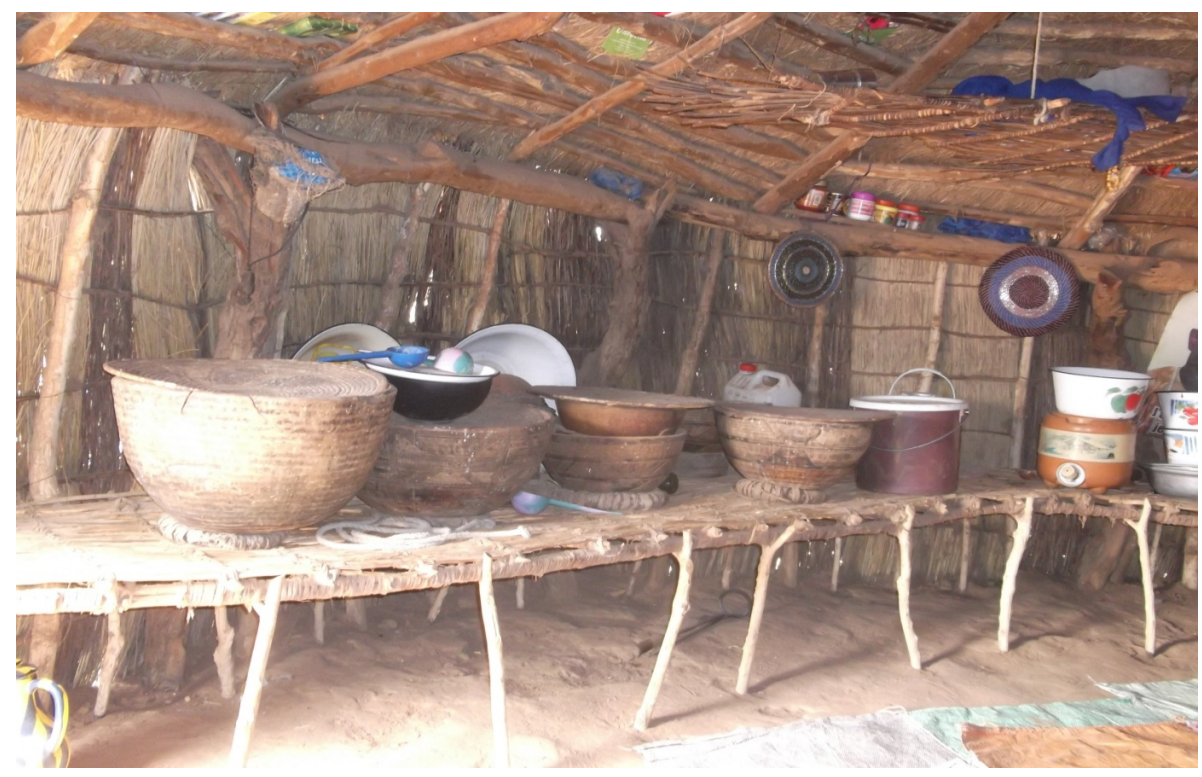

Sans vouloir revenir sur le rituel de fabrication du kaggu et ses pouvoirs magico-religieux déjà développés par Sada-Mamadou $\mathrm{Ba}^{22}$, il nous semble intéressant de souligner quelques pratiques, observées sur notre terrain, présentant certaines similitudes avec des pratiques du judaïsme. Sur le kaggu, il est strictement interdit de mélanger les calebasses de lait et les ustensiles de cuisine. D’après nos interlocuteurs, le mélange de ces différents objets peut occasionner un malheur tel que la perte du bétail. Cette séparation des objets contenant le lait de ceux servant à la cuisson des aliments n'est pas sans rappeler l'interdit fait aux Juifs : «tu ne cuiras pas le chevreau dans le lait de sa mère ». Selon Sophie Nizard (2012), le Talmud ${ }^{23}$ le comprend de manière très large : le chevreau, objet de cuisson, se réfère à la viande qui provient des mammifères domestiques permis. Par extension, le gibier et la volaille sont également concernés mais pas les poissons. Quant à l'acte de cuire ensemble laitage et viande, il s'étend à l'acte de manger au cours d'un même repas les deux types d'aliments. Sophie Nizard précise que les rédacteurs du Talmud ont encore étendu ces lois aux contenants. Ainsi vaisselles et ustensiles ayant été en contact avec des aliments carnés ou lactés, réputés absorber puis dégager d'infimes quantités d'aliments au cours de la cuisson, doivent être

\footnotetext{
${ }^{22}$ S.-M. BA, (2009), «Entre les pasteurs peuls et leurs vaches : une "parenté de lait" », article disponible sur: http://www.lemangeur-ocha.com/wp-content/uploads/2012/04/Le-lait-desPeuls.pdf, consulté le 22 octobre 2013.

${ }^{23}$ Le Talmud est l'ensemble des recueils rassemblant la loi orale (Tora) et ses commentaires.
} 
strictement séparés. Les Peuls du Ferlo, comme les Juifs observants ${ }^{24}$, évitent soigneusement de mélanger les ustensiles pour le lait et ceux destinés aux plats cuisinés ou ayant contenu des produits carnés. Mais les Peuls ne s'interdisent pas de préparer et d'ingérer ensemble nourritures carnées et lactées durant les repas, car le beurre de lait (nébam sirmé) est très présent dans les plats cuisinés. D’après un récit légendaire recueilli au Ferlo lors de nos enquêtes de terrain, les Peuls vivaient à l'origine en Inde avant de migrer vers l'Égypte ${ }^{25}$. Leur roi s'appelait Diéri ${ }^{26}$. Ils vénéraient un bœuf d'or qu'ils nommaient Toro. Un jour le prophète Moïse est venu prêcher la Parole de Dieu. Le roi Diéri dit aux Peuls : « moi je m’en vais. Même si vous suivez Moïse, ne vous séparez jamais de Toro. Il est le garant de votre unité ». Les Peuls furent convertis par Moïse, et Toro découpé en tas. Chaque famille prit sa part d'or. À son retour, Diéri constata que son conseil n'avait pas été respecté. Il sanglota et dit, «les Peuls ne seront plus unis jusqu'à la fin du monde ». D'après les Peuls du Ferlo, c'est depuis ce jour funeste qu'ils se sont dispersés à travers le monde ${ }^{27}$. Ceux qui sont arrivés jusqu'aux rives du fleuve Sénégal avec leurs bovins, ovins et caprins, s'implantèrent sur les rives sud du fleuve pour profiter de l'abondance de ses eaux et des pâturages environnants. De là, à chaque saison des pluies, des groupes d'hommes menaient les troupeaux dans les hautes terres du Ferlo. Après avoir apprivoisé cette nature, si ce n'est hostile, du moins contraignante, certains groupes peuls décidèrent de s'implanter de façon plus marquée et plus longue dans le Ferlo. Ils sont alors venus avec leurs familles et ont construit des campements constitués d'un ensemble de cases en paille appelées roumano, ce qui veut dire littéralement en langue peule « là où on passe la saison des pluies ».

\section{Conclusion}

Malgré des transformations profondes en cours, les Peuls du Ferlo ont préservé bon nombre d'habitudes et d'interdits alimentaires. L'organisation genrée des activités culinaires est légitimée par une myriade de normes intériorisées dès le plus jeune âge, mais qui ont tendance à s'estomper avec le retour des jeunes migrants animés d'un fort désir de « modernité ». Cette société pastorale est, malgré les apparences et les idées reçues (au sein même de la société sénégalaise), constamment en transformation grâce à la globalisation et aux migrations. Cette circulation d'idées et de personnes, qu'Arjun

\footnotetext{
${ }^{24}$ De nombreuses études ont démontré l'existence de ressemblances culturelles entre les Peuls et les Juifs à l'instar des travaux d'Henri Lhote (1958) et de Maurice Delafosse (1972).

${ }_{25}$ Dans De l'origine égyptienne des Peuls, I'historien Aboubacry-Moussa Lam (1993) revient sur la question controversée de l'origine de ces pasteurs supposés venir de l'Orient.

${ }^{26}$ Diéri est un prénom masculin que l'on retrouve chez les Peuls du Sénégal. Le terme désigne également une zone sableuse interstitielle entre la vallée du fleuve Sénégal et le Ferlo, habitée par des Peuls.

${ }^{27}$ Selon Maurice Delafosse (1972), le terme fulbé signifie « éparpillés ».
} 
Appadurai (1996) qualifie de flux, participe considérablement au façonnement des nouvelles manières de faire et de penser l'alimentation dans le Ferlo.

L'étude des restrictions et interdits alimentaires a permis de mettre en évidence l'affirmation identitaire des Peuls du Ferlo ainsi que les permanences et les transformations sociales. Cette volonté de démarcation vis-à-vis des autres communautés est soustendue par un soubassement moral et idéologique. Par une morale alimentaire ascétique et austère basée sur le respect des principes de la notion de "ndimaagu », les Peuls du Ferlo continuent à marquer leur différence culturelle. Cette position leur permet de valoriser l'image de l'endogroupe et de faire face à un milieu sahélien peu hospitalier (sécheresses récurrentes, dégradation de la nature à cause d'actions anthropiques). Le mode de vie de ces pasteurs est régi par un profond sentiment d'appartenance à un groupe plus ou moins uni autour d'un idéal commun, le pulaaku. Les contraintes liées au pastoralisme (mobilité, raréfaction des ressources, etc.) les obligent également à mener une vie rustique et frugale. Pour faire face aux changements sociaux et économiques actuels, la remobilisation de valeurs et pratiques anciennes a permis aux Peuls de se définir et de marquer leur identité face à une altérité changeante. Actuellement au Sénégal, des membres d'associations pour la promotion du pulaaku (Tabital pulaaku international, Dental université Cheikh Anta Diop, Kawral, Pinggal) sont très visibles dans les médias. Ce mouvement militant pour une préservation des valeurs culturelles peules est dirigé par des Peuls urbains instruits. Des radios comme Radio Fulbe et Tétiane sont spécialisées dans la promotion du pulaar, la langue peule. Des émissions en pulaar sont diffusées également sur certaines chaines de télévision sénégalaises (2STV, RDV, RTS). Ces exemples indiquent, à l'instar de ce que nous avons détaillé concernant les pratiques alimentaires dans le Ferlo, que la tradition et la modernité ne s'excluent aucunement au Sénégal, mais qu'elles se réinventent toutes deux pour en constituer le présent.

\section{Bibliographie}

Appadurai A. (1996), Modernity at Large: Cultural Dimensions of Globalization, Minneapolis, University of Minnesota Press.

BA A. H., Dieterlen G. (1966), «Les fresques d’époque bovidienne du Tassili N’Ajjer et les traditions des Peuls : hypothèses d'interprétation, Journal des Africanistes, $n^{\circ} 36$, p. 141-157.

BA A. H. (1991), Amkoullel, l'enfant peul, Paris, Éditions Actes Sud.

BARTH F. (1969), « Les groupes ethniques et leurs frontières », in P. PoutignAT, J. STREIFFFenart, Théories de l'ethnicité, Paris, Presses Universitaires de France, p. 203-249.

BiLlEn L. (2014), Des jardins féminins à l'ombre de la Grande Muraille Verte, Mémoire de Master 1, Lyon, ENS Lyon. 
BoCQUenne H. (1986), Moi, un Mbororo, Paris, Éditions Karthala.

Bollig M., Schulte A. (1999), «Environmental Change and Pastoral Perceptions: Degradation and Indigenous Knowledge in Two African Pastoral Communities », $\mathrm{Hu}$ man Ecology, vol. 27, $\mathrm{n}^{\circ}$ 3, p. 493-514.

BREEDVELD A., BRUIJN M. DE (1996), «L'image des Foulbé : Analyse critique de la construction du concept de pulaaku », Cahiers d'études africaines, $\mathrm{n}^{\circ}$ 144, p. 791-821.

CAMARA A. S. D. (2013), Le rapport au changement en société pastorale. Le cas des éleveurs du Ferlo et de Colonnat, Thèse de doctorat, Dijon, Université de Bourgogne.

Combessie J-C. (2007), La méthode en sociologie, Paris, La Découverte.

CRENN C, KA A. (2012), « "En quête” d'alimentation dans la zone Téssékéré/Widou au Sénégal », Les Cahiers de l'Observatoire International Homme-Milieux Téssékéré, $\mathrm{n}^{\circ} 2$, p. 37-48.

Delafosse M. (1972), Haut-Sénégal-Niger, Paris, G.P. Maisonneuve et Larose.

Demante M-J. (2006), Appui à la capitalisation des expériences du Projet d'Autopromotion Pastorale dans le Ferlo, Saint-Louis, PAPF.

Diop C. A. (1954), Nations nègres et culture, Paris, Présence Africaine.

Douglas M. (1971), De la souillure : essai sur les notions de pollution et de tabou, Paris, Éditions Maspero.

Droy I. (2011), « Le coton bio-équitable au Mali : un facteur de transformation sociale pour les femmes?», Mondes en développement, vol. 156, $\mathrm{n}^{\circ}$ 4, p. 47-58.

Dupire M. (1970), Organisation sociale des Peul. Étude d'ethnographie comparée, Paris, Éditions Plon.

FARB P., ARmelagos G. (1980), Anthropologie des coutumes alimentaires, Paris, Éditions Denoël.

FISCHLER C. (2001), L’Homnivore, Paris, Éditions Odile Jacob.

GARINE I. DE. (1962), « Usages alimentaires dans la région de Khombole (Sénégal) », Cahiers d'études africaines, $\mathrm{n}^{\circ} 10, \mathrm{p} .218-265$.

Giard L. (1994), «Faire la cuisine », in M. DE Certeau, L. Giard, P. Mayol, L'invention du quotidien, Paris, Gallimard, p. 213-314.

Gorre J.-P. (1996), « Hiérarchie sociale par le partage de la «boule » chez les Foulbé de Katako (Cameroun) », in A. Froment, I. DE GARINE, C. BinAm BiKoI, J.-F. Loung (dir.), Bien manger et bien vivre. Anthropologie alimentaire et développement en Afrique en Afrique intertropicale: du biologique au social, Paris, L'Harmattan, p. 393- 400.

Grenier P. (1960), Les Peul du Ferlo, Paris, Les Cahiers d'Outre-mer.

Guilhem D. (2012), « Peuls », in J.-P. Poulain (dir.), Dictionnaire des cultures alimentaires, Paris, PUF, p. 1011-1115. 
KA A. (2013), « Le jardin polyvalent de Widou Thiengoly : une organisation de femmes connectée à un réseau globalisé », Les Cahiers de l'Observateur International HommeMilieux Téssékéré, $\mathrm{n}^{\circ}$ 3, p. 57-63.

KA A. (2016), Manger à Widou Thiengoly (Nord-Sénégal): de l'abondance remémorée à la dépendance au marché, Thèse de doctorat, Dakar, Université Cheikh Anta Diop.

LABATUT R. (1973), Le parler d'un groupe de Peuls nomades : les Wodaabe Hoorewaalde Dageejabibbe Siroma (Nord-Cameroun), Paris, SELAF.

Lam A.-M. (1993), De l'origine égyptienne des Peuls, Paris, Présence africaine.

LeVI-STRauss C. (1962), La pensée sauvage, Paris, Éditions Plon.

Lhote H. (1958), L'extraordinaire aventure dès Peuls, Paris, Présence Africaine.

Ly B. (1966), L'Honneur et les valeurs morales dans les sociétés ouolof et toucouleur du Sénégal. Étude de sociologie, Thèse de $3^{\mathrm{e}}$ cycle, Paris, Université de Paris I.

MANOLI C., ANCEY V. (2013), « Effets ambigus de la mobilité des jeunes pasteurs : entre la sécurisation des conditions de vie pastorales et l'émancipation individuelle au Ferlo, Sénégal », in G. Azoulay, V. Ancey, D. Dormoy, C. Crenn, A. MAngu, A. ThomasHAUSEN (dir.). Mobilités et migrations : Figures et enjeux contemporains. Pluralité des regards et des disciplines, Paris, L’Harmattan, p. 185-201.

MEAd M. (1937), « Tabu », Encyclopedia of the Social Sciences, vol. 5, New York, Edwin R. A. Seligman and Alvin Johnson, p. 502-505.

Michaud P., SALL N. (2005), « L'éducation et la technologie : perspectives des femmes sénégalaises », Canadian Journal of Development Studies, vol. 26, nº 1, p. 107-130.

NizARD S. (2012), « Cacherout », in J.-P. Poulain, Dictionnaire des cultures alimentaires, Paris, Presses Universitaires de France, p. 221-226.

Poulain J.-P. (2002), Sociologie de l'alimentation, Paris, PUF.

RABAIN-JAmin J. (2003), « Enfance, âge et développement chez les Wolof du Sénégal », L'Homme, $\mathrm{n}^{\circ}$ 167, p. 49-66.

Radcliffe-Brown A R. (1939), Taboo, Cambridge, Editions Cambridge University Press.

RichaRdS A. (1932), Hunger and Work in a Savage Tribe. A functional study of nutrition among the Southern Bantu, Londres, Routledge.

Schmitz J. (1990), «Les Peul : Islam, pastoralisme et fluctuations du peuplement », Cahiers des Sciences Humaines, $\mathrm{n}^{\circ}$ 26, p. 499-504.

SERra MALlOl C. (2012a), « Une approche diachronique de l'alimentation à Tahiti », Bulletin de l'Association des Historiens et Géographes de Polynésie française, $\mathrm{n}^{\circ} 12$, novembre, p. 196-217.

Serra Mallol C. (2012b), « Tabou en Polynésie », in J.-P. POULAIN, Dictionnaire des cultures alimentaires, Paris, Presses Universitaires de France, p. 1312-1317. 
Sow S. (2015), «La place de la retenue alimentaire dans le code des valeurs chez les Peuls ", Les excès du boire et du manger, communication orale lors de l'Université d'été organisée par l'IEHCA du 30 août au 06 septembre 2015, Université François Rabelais de Tours.

TOURE O. (1997), « La gestion des ressources naturelles en milieu pastoral. L'exemple du Ferlo sénégalais », in P. Tersiguel, C. Becker, Développement durable au Sahel, Paris, Éditions Karthala, p. 125-143.

YounT C. (2010), Tout a changé, saufle repas : Les habitudes alimentaires dakaroises face aux enjeux socio-économiques, Mémoire de Master II, Paris, École des Hautes Études en Sciences Sociales/Centre Edgar Morin.

Webster H. (1942), Taboo: A Sociological Study, California, Editions Stanford University Press.

ZEZE-BeKe P. (1989), « Les interdits alimentaires chez les Nyabwa de Côte-d'Ivoire », Journal des Africanistes, $\mathrm{n}^{\circ}$ 59, p. 229-237. 